\title{
Continuity of the quenching time in a semilinear parabolic equation
}

\footnotetext{
ABSTRACT. In this paper, we consider the following initial-boundary value problem

$$
\left\{\begin{array}{l}
u_{t}=\Delta u-u^{-p} \text { in } \Omega \times(0, T), \\
\frac{\partial u}{\partial \nu}=0 \text { on } \partial \Omega \times(0, T), \\
u(x, 0)=u_{0}(x) \text { in } \bar{\Omega},
\end{array}\right.
$$

where $\Omega$ is a bounded domain in $\mathbb{R}^{N}$ with smooth boundary $\partial \Omega, p>0, \Delta$ is the Laplacian, $\nu$ is the exterior normal unit vector on $\partial \Omega$. Under some assumptions, we show that the solution of the above problem quenches in a finite time and estimate its quenching time. We also prove the continuity of the quenching time as a function of the initial data $u_{0}$. Finally, we give some numerical results to illustrate our analysis.
}

1. Introduction. Let $\Omega$ be a bounded domain in $\mathbb{R}^{N}$ with smooth boundary $\partial \Omega$. Consider the following initial-boundary value problem

$$
\begin{gathered}
u_{t}=\Delta u-u^{-p} \quad \text { in } \Omega \times(0, T), \\
\frac{\partial u}{\partial \nu}=0 \quad \text { on } \quad \partial \Omega \times(0, T), \\
u(x, 0)=u_{0}(x)>0 \text { in } \bar{\Omega},
\end{gathered}
$$

2000 Mathematics Subject Classification. 35B40, 35B50, 35K60, 65M06.

Key words and phrases. Quenching, nonlinear parabolic equation, numerical quenching time. 
where $p>0, \Delta$ is the Laplacian, $\nu$ is the exterior normal unit vector on $\partial \Omega$. The initial data $u_{0} \in C^{1}(\bar{\Omega})$ and satisfies the compatibility conditions. Here $(0, T)$ is the maximal time interval of existence of the solution $u$. The time $T$ may be finite or infinite. When $T$ is infinite, we say that the solution $u$ exists globally. When $T$ is finite, the solution $u$ develops a singularity in a finite time, namely

$$
\lim _{t \rightarrow T} u_{\min }(t)=0
$$

where $u_{\min }(t)=\min _{x \in \bar{\Omega}} u(x, t)$. In this last case, we say that the solution $u$ quenches in a finite time and the time $T$ is called the quenching time of the solution $u$. Thus we have $u(x, t)>0$ in $\bar{\Omega} \times[0, T)$. Solutions of semilinear parabolic equations which quench in a finite time have been the subject of investigation of many authors (see [2]-[4], [6], [7], [10], [11], [13], [14], [20], [24], [25], [27]-[29] and the references cited therein). In particular, in [7], the problem (1)-(3) has been studied. The local in time existence of a classical solution has been proved and this solution is unique (see [7]). It is also shown that the solution of (1)-(3) quenches in a finite time and its quenching time has been estimated (see [7]). In this paper, we are interested in the continuity of the quenching time as a function of the initial data $u_{0}$. More precisely, we consider the following initial-boundary value problem

$$
\begin{gathered}
v_{t}=\Delta v-v^{-p} \quad \text { in } \Omega \times\left(0, T_{h}\right), \\
\frac{\partial v}{\partial \nu}=0 \quad \text { on } \quad \partial \Omega \times\left(0, T_{h}\right), \\
v(x, 0)=v_{0}(x)>0 \quad \text { in } \bar{\Omega},
\end{gathered}
$$

where $v_{0}(x)=u_{0}(x)+h(x), h \in C^{1}(\bar{\Omega}), \frac{\partial h}{\partial \nu}=0$ on $\partial \Omega, h(x) \geq 0$ in $\bar{\Omega}$. Here $\left(0, T_{h}\right)$ is the maximal time interval on which the solution $v$ of (4)-(6) exists. When $T_{h}$ is finite, we say that the solution $v$ of (4)-(6) quenches in a finite time and the time $T_{h}$ is called the quenching time of the solution $v$. From the maximum principle, we have $v \geq u$ as long as all of them are defined. We deduce that $T_{h} \geq T$. In the present paper, we prove that if $\|h\|_{\infty}$ is small enough, then the solution $v$ of (4)-(6) quenches in a finite time and its quenching time $T_{h}$ goes to $T$ as $\|h\|_{\infty}$ goes to zero where $T$ is the quenching time of the solution $u$ of (1)-(3) and $\|h\|_{\infty}=\sup _{x \in \bar{\Omega}}|h(x)|$. Similar results have been obtained in [5], [8], [16], [19], [18], [21]-[23], [30] where the authors have considered the phenomenon of blow-up (we say that a solution blows up in a finite time if it reaches the value infinity in a finite time). The rest of the paper is organized as follows. In the next section, under some assumptions, we show that the solution $v$ of (4)-(6) quenches in a finite time and estimate its quenching time. In the third section, we prove the continuity of the quenching time and finally in the last section, we give some numerical results to illustrate our analysis. 
2. Quenching time. In this section, under some assumptions, we show that the solution $v$ of (4)-(6) quenches in a finite time and estimate its quenching time.

Using an idea of Friedman and McLeod in [15], we may prove the following result.

Theorem 2.1. Suppose that there exists a constant $A \in(0,1]$ such that the initial data at (6) satisfies

$$
\Delta v_{0}(x)-v_{0}^{-p}(x) \leq-A v_{0}^{-p}(x) \text { in } \Omega .
$$

Then, the solution $v$ of (4)-(6) quenches in a finite time $T_{h}$ which obeys the following estimate

$$
T_{h} \leq \frac{\left\|v_{0}\right\|_{\mathrm{inf}}^{p+1}}{A(p+1)},
$$

where $\left\|v_{0}\right\|_{\text {inf }}=\min _{x \in \bar{\Omega}} v_{0}(x)$.

Proof. Since $\left(0, T_{h}\right)$ is the maximal time interval of existence of the solution $v$, our aim is to show that $T_{h}$ is finite and satisfies the above inequality. Introduce the function $J(x, t)$ defined as follows

$$
J(x, t)=v_{t}(x, t)+A v^{-p}(x, t) \text { in } \bar{\Omega} \times\left(0, T_{h}\right) .
$$

A straightforward computation yields

$$
J_{t}-\Delta J=\left(v_{t}-\Delta v\right)_{t}-A p v^{-p-1} v_{t}-A \Delta v^{-p} \text { in } \Omega \times\left[0, T_{h}\right) .
$$

By a direct calculation, we observe that

$$
\Delta v^{-p}=p(p+1) v^{-p-2}|\nabla v|^{2}-p v^{-p-1} \Delta v,
$$

which implies that $\Delta v^{-p} \geq-p v^{-p-1} \Delta v$. Using this estimate and (8), we arrive at

$$
J_{t}-\Delta J \leq\left(v_{t}-\Delta v\right)_{t}-A p v^{-p-1}\left(v_{t}-\Delta v\right) \text { in } \Omega \times\left(0, T_{h}\right) .
$$

It follows from (4) and (9) that

$$
J_{t}-\Delta J \leq p v^{-p-1} v_{t}+A p v^{-2 p-1} \quad \text { in } \Omega \times\left(0, T_{h}\right) .
$$

Taking into account the expression of $J$, we find that

$$
J_{t}-\Delta J \leq p v^{-p-1} J \text { in } \Omega \times\left(0, T_{h}\right) .
$$

We also have

$$
\frac{\partial J}{\partial \nu}=\left(\frac{\partial v}{\partial \nu}\right)_{t}-A p v^{-p-1} \frac{\partial v}{\partial \nu}=0 \quad \text { on } \quad \partial \Omega \times\left(0, T_{h}\right)
$$

and due to (7), we discover that

$$
J(x, 0)=\Delta v_{0}(x)-v_{0}^{-p}(x)+A v_{0}^{-p}(x) \leq 0 \quad \text { in } \quad \Omega .
$$

From the maximum principle, we have

$$
J(x, t) \leq 0 \quad \text { in } \Omega \times\left(0, T_{h}\right),
$$


which implies that

$$
v_{t}(x, t)+A v^{-p}(x, t) \leq 0 \quad \text { in } \quad \Omega \times\left(0, T_{h}\right) .
$$

This estimate may be rewritten in the following manner

$$
v^{p} d v \leq-A d t \quad \text { in } \Omega \times\left(0, T_{h}\right) .
$$

Integrate the above inequality over $\left(0, T_{h}\right)$ to obtain

$$
T_{h} \leq \frac{(v(x, 0))^{p+1}}{A(p+1)} \quad \text { for } \quad x \in \Omega .
$$

We deduce that

$$
T_{h} \leq \frac{\left\|v_{0}\right\|_{i n f}^{p+1}}{A(p+1)} .
$$

Consequently, $v$ quenches at the time $T_{h}$ because the quantity on the right hand side of the above inequality is finite and the proof is complete.

Remark 2.1. Let $t_{0} \in\left(0, T_{h}\right)$. Integrating the inequality in (10) from $t_{0}$ to $T_{h}$, we get

$$
T_{h}-t_{0} \leq \frac{\left(v\left(x, t_{0}\right)\right)^{p+1}}{A(p+1)} \text { for } \quad x \in \Omega
$$

We deduce that

$$
T_{h}-t_{0} \leq \frac{\left(v_{\min }\left(t_{0}\right)\right)^{p+1}}{A(p+1)}
$$

3. Continuity of the quenching time. In this section, under some assumptions, we show that the solution $v$ of (4)-(6) quenches in a finite time and its quenching time goes to that of the solution $u$ of (1)-(3) when $\|h\|_{\infty}$ goes to zero.

Firstly, we show that the solution $v$ approaches the solution $u$ in $\bar{\Omega} \times$ $[0, T-\tau]$ with $\tau \in(0, T)$ when $\|h\|_{\infty}$ tends to zero. This result is stated in the following theorem.

Theorem 3.1. Let $u$ be the solution of (1)-(3). Suppose that $u \in C^{2,1}(\bar{\Omega} \times$ $[0, T-\tau])$ and $\min _{t \in[0, T-\tau]} u_{\min }(t)=\alpha>0$ with $\tau \in(0, T)$. Then, the problem (4)-(6) admits a unique solution $v \in C^{2,1}\left(\bar{\Omega} \times\left[0, T_{h}\right)\right)$ and the following relation holds

$$
\sup _{t \in[0, T-\tau]}\|v(\cdot, t)-u(\cdot, t)\|_{\infty}=0\left(\|h\|_{\infty}\right) \quad \text { as } \quad\|h\|_{\infty} \rightarrow 0 .
$$

Proof. The problem (4)-(6) has for each $h$, a unique solution $v \in C^{2,1}(\bar{\Omega} \times$ $\left.\left[0, T_{h}\right)\right)$. In the introduction of the paper, we have seen that $T_{h} \geq T$. Let $t(h) \leq T$ the greatest value of $t>0$ such that

$$
\|v(\cdot, t)-u(\cdot, t)\|_{\infty} \leq \frac{\alpha}{2} \quad \text { for } \quad t \in(0, t(h)) .
$$


By a direct calculation, we see that $\|v(\cdot, 0)-u(\cdot, 0)\|_{\infty}=\left\|v_{0}-u_{0}\right\|_{\infty}=\|h\|_{\infty}$, which implies that $\|v(\cdot, 0)-u(\cdot, 0)\|_{\infty}$ tends to zero as $\|h\|_{\infty}$ goes to zero. Due to this fact, we deduce that $t(h)>0$ for $\|h\|_{\infty}$ sufficiently small. By the triangle inequality, we obtain

$$
v_{\min }(t) \geq u_{\min }(t)-\|v(\cdot, t)-u(\cdot, t)\|_{\infty} \quad \text { for } \quad t \in(0, t(h)),
$$

which leads us to

$$
v_{\min }(t) \geq \alpha-\frac{\alpha}{2}=\frac{\alpha}{2} \quad \text { for } \quad t \in(0, t(h)) .
$$

Introduce the function $e(x, t)$ defined as follows

$$
e(x, t)=v(x, t)-u(x, t) \text { in } \bar{\Omega} \times[0, t(h)) .
$$

A routine computation reveals that

$$
\begin{gathered}
e_{t}-\Delta e=p \theta^{-p-1} e \quad \text { in } \quad \Omega \times(0, t(h)), \\
\frac{\partial e}{\partial \nu}=0 \quad \text { on } \quad \partial \Omega \times(0, t(h)), \\
e(x, 0) \geq h(x) \quad \text { in } \Omega,
\end{gathered}
$$

where $\theta$ is an intermediate value between $u$ and $v$. Let

$$
z(x, t)=e^{(L+1) t}\|h\|_{\infty} \quad \text { in } \bar{\Omega} \times[0, T],
$$

where $L=p\left(\frac{\alpha}{2}\right)^{-p-1}$. It is not hard to see that $L=p\left(\frac{\alpha}{2}\right)^{-p-1} \geq p \theta^{-p-1}$. Thanks to this observation, a straightforward calculation yields

$$
\begin{gathered}
z_{t}-\Delta z \geq p \theta^{-p-1} z \quad \text { in } \quad \Omega \times(0, t(h)), \\
\frac{\partial z}{\partial \nu}=0 \quad \text { on } \quad \partial \Omega \times(0, t(h)), \\
z(x, 0) \geq e(x, 0) \quad \text { in } \Omega .
\end{gathered}
$$

It follows from the maximum principle that

$$
z(x, t) \geq e(x, t) \quad \text { in } \Omega \times(0, t(h)) .
$$

By the same way, we also prove that

$$
z(x, t) \geq-e(x, t) \quad \text { in } \Omega \times(0, t(h)),
$$

which implies that

$$
\|e(\cdot, t)\|_{\infty} \leq e^{(L+1) t}\|h\|_{\infty} \quad \text { for } \quad t \in(0, t(h)) .
$$

Let us show that $t(h)=T$. Suppose that $t(h)<T$. From (13), we obtain

$$
\frac{\alpha}{2}=\|v(\cdot, t(h))-u(\cdot, t(h))\|_{\infty} \leq e^{(L+1) T}\|h\|_{\infty} .
$$

Since the term on the right hand side of the above inequality goes to zero as $\|h\|_{\infty}$ goes to zero, we deduce that $\frac{\alpha}{2} \leq 0$, which is impossible. Consequently, $t(h)=T$ and the proof is complete. 
Now, we are in a position to prove the main result of the paper.

Theorem 3.2. Suppose that the problem (1)-(3) has a solution $u$ which quenches at the time $T$ and $u \in C^{2,1}(\bar{\Omega} \times[0, T))$. Under the assumption of Theorem 2.1, the problem (4)-(6) has a solution $v$ which quenches in a finite time $T_{h}$ and the following relation holds

$$
\lim _{\|h\|_{\infty} \rightarrow 0} T_{h}=T \text {. }
$$

Proof. Let $\varepsilon>0$. There exists $\rho>0$ such that

$$
\frac{y^{p+1}}{A(p+1)} \leq \frac{\varepsilon}{2}, \quad 0 \leq y \leq \rho .
$$

Since $u$ quenches in a finite time $T$, there exists $T_{0} \in\left(T-\frac{\varepsilon}{2}, T\right)$ such that

$$
0<u_{\min }(t)<\frac{\rho}{2} \text { for } t \in\left[T_{0}, T\right) .
$$

Set $T_{1}=\frac{T_{0}+T}{2}$. It is not hard to see that

$$
u_{\min }(t)>0 \text { for } t \in\left[0, T_{1}\right] .
$$

From Theorem 3.1, the problem (4)-(6) has a solution $v$ and we get

$$
\|v(\cdot, t)-u(\cdot, t)\|_{\infty}<\frac{\rho}{2} \quad \text { for } t \in\left[0, T_{1}\right],
$$

which implies that $\left\|v\left(\cdot, T_{1}\right)-u\left(\cdot, T_{1}\right)\right\|_{\infty} \leq \frac{\rho}{2}$. An application of the triangle inequality leads us to

$$
v_{\min }\left(T_{1}\right) \leq\left\|v\left(\cdot, T_{1}\right)-u\left(\cdot, T_{1}\right)\right\|_{\infty}+u_{\min }\left(T_{1}\right) \leq \frac{\rho}{2}+\frac{\rho}{2}=\rho .
$$

From Theorem 2.1, $v$ quenches at the time $T_{h}$. We deduce from Remark 2.1 and (14) that

$$
0 \leq T_{h}-T=T_{h}-T_{1}+T_{1}-T \leq \frac{\left(v_{\min }\left(T_{1}\right)\right)^{p+1}}{A(p+1)}+\frac{\varepsilon}{2} \leq \varepsilon,
$$

and the proof is complete.

4. Numerical results. In this section, we give some computational experiments to confirm the theory given in the previous section. We consider the radial symmetric solution of the following initial-boundary value problem

$$
\begin{gathered}
u_{t}=\Delta u-u^{-p} \quad \text { in } \quad B \times(0, T), \\
\frac{\partial u}{\partial \nu}=0 \quad \text { on } \quad S \times(0, T), \\
u(x, 0)=u_{0}(x) \text { in } B,
\end{gathered}
$$

where $B=\left\{x \in \mathbb{R}^{N} ;\|x\|<1\right\}, S=\left\{x \in \mathbb{R}^{N} ;\|x\|=1\right\}$. The above problem may be rewritten in the following form 


$$
\begin{gathered}
u_{t}=u_{r r}+\frac{N-1}{r} u_{r}-u^{-p}, \quad r \in(0,1), \quad t \in(0, T), \\
u_{r}(0, t)=0, \quad u_{r}(1, t)=0, \quad t \in(0, T), \\
u(r, 0)=\varphi(r), \quad r \in(0,1) .
\end{gathered}
$$

Here, we take $\varphi(r)=\frac{2+\cos (\pi r)}{4}+\varepsilon(1+\cos (\pi r))$ where $\varepsilon \in[0,1]$. We start with the construction of an adaptive scheme as follows. Let $I$ be a positive integer and let $h=1 / I$. Define the grid $x_{i}=i h, 0 \leq i \leq I$ and approximate the solution $u$ of (15)-(17) by the solution $U_{h}^{(n)}=\left(U_{0}^{(n)}, \ldots, U_{I}^{(n)}\right)^{T}$ of the following explicit scheme

$$
\begin{aligned}
\frac{U_{0}^{(n+1)}-U_{0}^{(n)}}{\Delta t_{n}}= & N \frac{2 U_{1}^{(n)}-2 U_{0}^{(n)}}{h^{2}}-\left(U_{0}^{(n)}\right)^{-p-1} U_{0}^{(n+1)}, \\
\frac{U_{i}^{(n+1)}-U_{i}^{(n)}}{\Delta t_{n}}= & \frac{U_{i+1}^{(n)}-2 U_{i}^{(n)}+U_{i-1}^{(n)}}{h^{2}}+\frac{(N-1)}{i h} \frac{U_{i+1}^{(n)}-U_{i-1}^{(n)}}{2 h} \\
& -\left(U_{i}^{(n)}\right)^{-p-1} U_{i}^{(n+1)}, \quad 1 \leq i \leq I-1, \\
\frac{U_{I}^{(n+1)}-U_{I}^{(n)}}{\Delta t_{n}}= & N \frac{2 U_{I-1}^{(n)}-2 U_{I}^{(n)}}{h^{2}}-\left(U_{I}^{(n)}\right)^{-p-1} U_{I}^{(n+1)}, \\
U_{i}^{(0)}= & \varphi_{i}, \quad 0 \leq i \leq I,
\end{aligned}
$$

where $\Delta t_{n}=\min \left\{\frac{\left(1-h^{2}\right) h^{2}}{2 N}, h^{2}\left\|U_{h}^{(n)}\right\|_{\text {inf }}^{p+1}\right\}$ with $\left\|U_{h}^{(n)}\right\|_{\text {inf }}=\min _{0 \leq i \leq I} U_{i}^{(n)}$. Let us notice that the restriction on the time step ensures the positivity of the discrete solution. We also approximate the solution $u$ of (15)-(17) by the solution $U_{h}^{(n)}$ of the implicit scheme below

$$
\begin{aligned}
\frac{U_{0}^{(n+1)}-U_{0}^{(n)}}{\Delta t_{n}}= & N \frac{2 U_{1}^{(n+1)}-2 U_{0}^{(n+1)}}{h^{2}}-\left(U_{0}^{(n)}\right)^{-p-1} U_{0}^{(n+1)} \\
\frac{U_{i}^{(n+1)}-U_{i}^{(n)}}{\Delta t_{n}}= & \frac{U_{i+1}^{(n+1)}-2 U_{i}^{(n+1)}+U_{i-1}^{(n+1)}}{h^{2}}+\frac{(N-1)}{i h} \frac{U_{i+1}^{(n+1)}-U_{i-1}^{(n+1)}}{2 h} \\
& -\left(U_{i}^{(n)}\right)^{-p-1} U_{i}^{(n+1)}, \quad 1 \leq i \leq I-1, \\
\frac{U_{I}^{(n+1)}-U_{I}^{(n)}}{\Delta t_{n}}= & N \frac{2 U_{I-1}^{(n+1)}-2 U_{I}^{(n+1)}}{h^{2}}-\left(U_{I}^{(n)}\right)^{-p-1} U_{I}^{(n+1)}, \\
U_{i}^{(0)}= & \varphi_{i}, \quad 0 \leq i \leq I,
\end{aligned}
$$

where $\Delta t_{n}=h^{2}\left\|U_{h}^{(n)}\right\|_{\text {inf }}^{p+1}$.

Let us again remark that for the above implicit scheme, the existence and positivity of the discrete solution is also guaranteed using standard methods 
(see for instance [6]). It is not hard to see that $u_{x x}(1, t)=\lim _{r \rightarrow 1} \frac{u_{r}(r, t)}{r}$ and $u_{x x}(0, t)=\lim _{r \rightarrow 0} \frac{u_{r}(r, t)}{r}$. Hence, if $r=0$ and $r=1$, we see that

$$
\begin{aligned}
& u_{t}(0, t)=N u_{r r}(0, t)-u^{-p}(0, t), \quad t \in(0, T), \\
& u_{t}(1, t)=N u_{r r}(1, t)-u^{-p}(1, t), \quad t \in(0, T) .
\end{aligned}
$$

These observations have been taken into account in the construction of our schemes when $i=0$ and $i=I$. We need the following definition.

Definition 4.1. We say that the discrete solution $U_{h}^{(n)}$ of the explicit scheme or the implicit scheme quenches in a finite time if

$$
\lim _{n \rightarrow \infty}\left\|U_{h}^{(n)}\right\|_{\text {inf }}=0
$$

and the series $\sum_{n=0}^{\infty} \Delta t_{n}$ converges. The quantity $\sum_{n=0}^{\infty} \Delta t_{n}$ is called the numerical quenching time of the discrete solution $U_{h}^{(n)}$.

In the following tables, in rows, we present the numerical quenching times, the numbers of iterations, the CPU times and the orders of the approximations corresponding to meshes of $16,32,64,128$. We take for the numerical quenching time $T^{n}=\sum_{j=0}^{n-1} \Delta t_{j}$ which is computed at the first time when

$$
\Delta t_{n}=\left|T^{n+1}-T^{n}\right| \leq 10^{-16} .
$$

The order(s) of the method is computed from

$$
s=\frac{\log \left(\left(T_{4 h}-T_{2 h}\right) /\left(T_{2 h}-T_{h}\right)\right)}{\log (2)} .
$$

\section{Numerical experiments for $p=1, N=2$.}

First case: $\varepsilon=0$.

Table 1: Numerical quenching times, numbers of iterations, CPU times (seconds) and orders of the approximations obtained with the explicit Euler method.

\begin{tabular}{|l|l|l|l|l|}
\hline $\mathrm{I}$ & $T^{n}$ & $\mathrm{n}$ & $C P U_{t}$ & $\mathrm{~s}$ \\
\hline 16 & 0.055728 & 1921 & 4 & - \\
\hline 32 & 0.054213 & 7048 & 27 & - \\
\hline 64 & 0.053488 & 25371 & 195 & 1.06 \\
\hline 128 & 0.053127 & 82924 & 1836 & 1.00 \\
\hline
\end{tabular}


Table 2: Numerical quenching times, numbers of iterations, CPU times (seconds) and orders of the approximations obtained with the first implicit Euler method.

\begin{tabular}{|l|l|l|l|l|}
\hline $\mathrm{I}$ & $T^{n}$ & $\mathrm{n}$ & $C P U_{t}$ & $\mathrm{~s}$ \\
\hline 16 & 0.054900 & 1908 & 5 & - \\
\hline 32 & 0.053847 & 7020 & 29 & - \\
\hline 64 & 0.053319 & 28007 & 429 & 1.00 \\
\hline 128 & 0.053111 & 87262 & 1965 & 0.99 \\
\hline
\end{tabular}

Second case: $\varepsilon=1 / 50$.

Table 3: Numerical quenching times, numbers of iterations, CPU times (seconds) and orders of the approximations obtained with the explicit Euler method.

\begin{tabular}{|l|l|l|l|l|}
\hline $\mathrm{I}$ & $T^{n}$ & $\mathrm{n}$ & $C P U_{t}$ & $\mathrm{~s}$ \\
\hline 16 & 0.058875 & 1946 & 5 & - \\
\hline 32 & 0.057148 & 7152 & 28 & - \\
\hline 64 & 0.056321 & 25803 & 215 & 0.88 \\
\hline 128 & 0.056012 & 102818 & 20237 & 0.82 \\
\hline
\end{tabular}

Table 4: Numerical quenching times, numbers of iterations, CPU times (seconds) and orders of the approximations obtained with the first implicit Euler method.

\begin{tabular}{|l|l|l|l|l|}
\hline $\mathrm{I}$ & $T^{n}$ & $\mathrm{n}$ & $C P U_{t}$ & $\mathrm{~s}$ \\
\hline 16 & 0.057270 & 1934 & 5 & - \\
\hline 32 & 0.055651 & 7100 & 30 & - \\
\hline 64 & 0.054877 & 25590 & 204 & 1.05 \\
\hline 128 & 0.054525 & 98037 & 1203 & 1.15 \\
\hline
\end{tabular}

Third case: $\varepsilon=1 / 100$.

Table 5: Numerical quenching times, numbers of iterations, CPU times (seconds) and orders of the approximations obtained with the explicit Euler method.

\begin{tabular}{|l|l|l|l|l|}
\hline $\mathrm{I}$ & $T^{n}$ & $\mathrm{n}$ & $C P U_{t}$ & $\mathrm{~s}$ \\
\hline 16 & 0.057270 & 1934 & 5 & - \\
\hline 32 & 0.055651 & 7100 & 30 & - \\
\hline 64 & 0.054877 & 25590 & 204 & 1.05 \\
\hline 128 & 0.054525 & 98037 & 1203 & 1.15 \\
\hline
\end{tabular}


Table 6: Numerical quenching times, numbers of iterations, CPU times (seconds) and orders of the approximations obtained with the first implicit Euler method.

\begin{tabular}{|l|l|l|l|l|}
\hline $\mathrm{I}$ & $T^{n}$ & $\mathrm{n}$ & $C P U_{t}$ & $\mathrm{~s}$ \\
\hline 16 & 0.056356 & 2009 & 6 & - \\
\hline 32 & 0.055248 & 7436 & 30 & - \\
\hline 64 & 0.054689 & 27070 & 328 & 0.98 \\
\hline 128 & 0.054426 & 98873 & 1318 & 1.08 \\
\hline
\end{tabular}

Remark 4.1. If we consider the problem (15)-(17) in the case where the initial data $\varphi(r)=\frac{2+\cos (\pi r)}{4}$ and $p=1$, we see that the numerical quenching time of the discrete solution for the explicit scheme or the implicit scheme is slightly equal 0.053 (see Tables 1 and 2). We observe from Tables 3, 4, 5 and 6 that if the above initial data increases slightly, then the numerical quenching time also increases slightly. This result confirms the theory established in the previous section.

\section{REFERENCES}

[1] Abia, L. M., López-Marcos, J. C. and Martínez, J., On the blow-up time convergence of semidiscretizations of reaction-diffusion equations, Appl. Numer. Math. 26 (1998), 399-414.

[2] Acker, A., Walter, W., The quenching problem for nonlinear parabolic differential equations, Ordinary and partial differential equations (Proc. Fourth Conf., Univ. Dundee, Dundee, 1976), Lecture Notes in Math., Vol. 564, Springer, Berlin, 1976, $1-12$.

[3] Acker, A., Kawohl, B., Remarks on quenching, Nonlinear Anal. 13 (1989), 53-61.

[4] Bandle, C., Braumer, C. M., Singular perturbation method in a parabolic problem with free boundary, BAIL IV (Novosibirsk, 1986), Boole Press Conf. Ser., 8, Boole, Dún Laoghaire, 1986, 7-14.

[5] Baras, P., Cohen, L., Complete blow-up after $T_{\max }$ for the solution of a semilinear heat equation, J. Funct. Anal. 71 (1987), 142-174.

[6] Boni, T. K., Extinction for discretizations of some semilinear parabolic equations, C. R. Acad. Sci. Paris Sér. I Math. 333 (2001), 795-800.

[7] Boni, T. K., On quenching of solutions for some semilinear parabolic equations of second order, Bull. Belg. Math. Soc. 7 (2000), 73-95.

[8] Cortazar, C., del Pino, M. and Elgueta, M., On the blow-up set for $u_{t}=\Delta u^{m}+u^{m}$, $m>1$, Indiana Univ. Math. J. 47 (1998), 541-561.

[9] Cortazar, C., del Pino, M. and Elgueta, M., Uniqueness and stability of regional blow-up in a porous-medium equation, Ann. Inst. H. Poincaré Anal. Non Linéare 19 (2002), 927-960.

[10] Deng, K., Levine, H. A, On the blow-up of $u_{t}$ at quenching, Proc. Amer. Math. Soc. 106 (1989), 1049-1056.

[11] Deng, K., Xu, M., Quenching for a nonlinear diffusion equation with singular boundary condition, Z. Angew. Math. Phys. 50 (1999), 574-584.

[12] Fermanian Kammerer, C., Merle, F. and Zaag, H., Stability of the blow-up profile of nonlinear heat equations from the dynamical system point of view, Math. Ann. 317 (2000), 195-237. 
[13] Fila, M., Kawohl, B. and Levine, H. A., Quenching for quasilinear equations, Comm. Partial Differential Equations 17 (1992), 593-614.

[14] Fila, M., Levine, H. A., Quenching on the boundary, Nonlinear Anal. 21 (1993), 795-802.

[15] Friedman, A., McLeod, B., Blow-up of positive solutions of nonlinear heat equations, Indiana Univ. Math. J., 34 (1985), 425-477.

[16] Galaktionov, V. A., Boundary value problems for the nonlinear parabolic equation $u_{t}=\Delta u^{\sigma+1}+u^{\beta}$, Differential Equations 17 (1981), 551-555.

[17] Galaktionov, V. A., Kurdjumov, S. P., Mihălov, A. P. and Samarskiu, A. A., On unbounded solutions of the Cauchy problem for the parabolic equation $u_{t}=$ $\nabla\left(u^{\sigma} \nabla u\right)+u^{\beta}$, (Russian) Dokl. Akad. Nauk SSSR 252 (1980), no. 6, 1362-1364.

[18] Galaktionov, V. A., Vazquez, J. L., Continuation of blow-up solutions of nonlinear heat equations in several space dimensions, Comm. Pure Appl. Math., 50 (1997), $1-67$.

[19] Galaktionov, V. A., Vazquez, J. L., The problem of blow-up in nonlinear parabolic equation, Current developments in partial differential equations (Temuco, 1999). Discrete Contin. Dyn. Syst. 8 (2002), 399-433.

[20] Guo, J., On a quenching problem with Robin boundary condition, Nonlinear Anal. 17 (1991), 803-809.

[21] Groisman, P., Rossi, J. D., Dependence of the blow-up time with respect to parameters and numerical approximations for a parabolic problem, Asymptot. Anal. 37 (2004), 79-91.

[22] Groisman, P., Rossi, J. D. and Zaag, H., On the dependence of the blow-up time with respect to the initial data in a semilinear parabolic problem, Comm. Partial Differential Equations 28 (2003), 737-744.

[23] Herrero, M. A., Velázquez, J. J. L., Generic behaviour of one-dimensional blow up patterns, Ann. Scuola Norm. Sup. Pisa Cl. Sci. (4) 19 (1992), no. 3, 381-450.

[24] Kawarada, H., On solutions of initial-boundary problem for $u_{t}=u_{x x}+1 /(1-u)$, Publ. Res. Inst. Math. Sci. 10 (1974/75), 729-736.

[25] Kirk, C. M., Roberts, C. A., A review of quenching results in the context of nonlinear Volterra equations, Dyn. Contin. Discrete Impuls. Syst. Ser. A Math. Anal., 10 (2003), 343-356.

[26] Ladyzenskaya, A., Solonnikov, V. A. and Ural'ceva, N. N., Linear and quasilinear equations parabolic type, Translations of Mathematical Monographs, Vol. 23, American Mathematical Society, Providence, R.I., 1967.

[27] Levine, H. A., The phenomenon of quenching: a survey, Trends in the theory and practice of nonlinear analysis (Arlington, Tex., 1984), North-Holland Math. Stud., 110, North-Holland, Amsterdam, 1985, 275-286.

[28] Levine, H. A., The quenching of solutions of linear parabolic and hyperbolic equations with nonlinear boundary conditions, SIAM J. Math. Anal. 14 (1983), 1139-1152.

[29] Levine, H. A., Quenching, nonquenching and beyond quenching for solution of some parabolic equations, Ann. Math. Pura Appl. (4) 155 (1989), 243-260.

[30] Merle, F., Solution of a nonlinear heat equation with arbitrarily given blow-up points, Comm. Pure Appl. Math. 45 (1992), 293-300.

[31] Nakagawa, T., Blowing up of the finite difference solution to $u_{t}=u_{x x}+u^{2}$, Appl. Math. Optim. 2 (1975/76), 337-350.

[32] Phillips, D., Existence of solution of quenching problems, Appl. Anal. 24 (1987), 253-264.

[33] Protter, M. H., Weinberger, H. F., Maximum Principles in Differential Equations, Prentice-Hall, Inc., Englewood Cliffs, N. J., 1967. 
[34] Quittner, P., Continuity of the blow-up time and a priori bounds for solutions in superlinear parabolic problems, Houston J. Math. 29 (2003), no. 3, 757-799 (electronic).

[35] Sheng, Q., Khaliq, A. Q. M., A compound adaptive approach to degenerate nonlinear quenching problems, Numer. Methods Partial Differential Equations, 15 (1999), 2947.

[36] Walter, W, Differential- und Integral-Ungleichungen und ihre Anwendung bei Abschätzungs- und Eindeutigkeits-problemen, (German) Springer Tracts in Natural Philosophy, Vol. 2, Springer-Verlag, Berlin-New York, 1964.

Théodore K. Boni

Institut National Polytechnique

Houphouët-Boigny de Yamoussoukro

BP 1093 Yamoussoukro

Côte d'Ivoire

e-mail: theokboni@yahoo.fr

Received April 11, 2008
Firmin K. N'gohisse

Université d'Abobo-Adjamé, UFR-SFA

Département de Mathmatiques et Informatiques 02 BP 801 Abidjan 02

Côte d'Ivoire

e-mail: firmingoh@yahoo.fr 